\title{
Micro-RNA in development
}

\author{
Thikekar V.P. ${ }^{1}$, Sawant J.J. ${ }^{2}$, More M.K. ${ }^{3}$, Nair V.K. ${ }^{4}$, Athalekar P. ${ }^{5}$, Patil D. ${ }^{6}$, Kulkarni S. ${ }^{7}$ and \\ Gomase V.S. ${ }^{8}$ \\ 'Bhartiya Gramin Punarrachana Sanstha's Arts, Commerce and Science, Wadala,Mumbai, India \\ ${ }^{2}$ Gogate Jogalekar College of Science, Arts and Commerce, Ratnagiri, India \\ ${ }^{3}$ Tilak College of Science and Commerce, Vashi, Navi Mumbai, India \\ ${ }^{4}$ Bhavan's College, Andheri (W), Mumbai- 400058, India \\ ${ }^{5}$ K.J. Somaiya College of Science and Commerce, Vidyavihar, Mumbai, India \\ ${ }^{6}$ Karmaveer Bhaurao Patil College, Vashi, Navi mumbai, India \\ ${ }^{7}$ Jaihind Educational Trust's- Z.B. Patil College, Dhule, India \\ ${ }^{8}$ School of Technology, S.R.T.M. University, Sub-Centre, Latur, 413512, India
}

\begin{abstract}
This article reviews the available literature on the regulation of miRNA processing that occurs within normal cells and embryonic stem cells, during development or in diseases such as cancer. Also, explains miRNA biogenesis process for which regulation occurs. RNA editing of miRNA precursors, regulation by RNA binding proteins, alterations in the levels of key processing proteins, as well as a number of unknown mechanisms contribute to the regulation of miRNA processing as well as miRNA expression pattern during adipogenesis.
\end{abstract}

Keywords- Micro-RNA, synaptic fractions, precursors, down regulation, adipogenesis, stem cell

\section{Introduction}

In 1933 Lee and colleagues first described function of miRNA to downregulate gene expression for C. elegans[1]. As of 2002, miRNAs have been confirmed in various plants and animals, including $C$. elegans, human and the plant Arabidopsis thaliana. MicroRNAs (miRNA or $\mu R N A$ ) are single-stranded RNA molecules of 21-23 nucleotides in length, which regulate gene expression. miRNAs are encoded by genes from whose DNA they are transcribed but miRNAs are not translated into protein.primary transcript is processed into a short stem-loop structure called a pre-miRNA and finally into a functional miRNA. MicroRNAs are produced from either their own genes or from introns and can be encoded by independent genes, but also be processed from a variety of different RNA species, including introns, 3' UTRs of mRNAs, long noncoding RNA, snoRNAs and transposons [2,3]. Recently, researchers found that miRNA genes overlap with the proteincoding region of the genes of a multigene family. Perfect or near perfect base pairing with the target RNA promotes cleavage of the RNA. In animals, microRNAs more often only partially base pair and inhibit protein translation of the target Mirna. MicroRNAs that are partially complementary to the target can also speed up deadenylation, causing mRNAs to be degraded sooner[4]. miRNAs occasionally also causes DNA methylation of promoter sites and therefore affecting the expression of targeted genes[5,6]. miRNAs function in association with a complement of proteins collectively termed the miRNP. Human miRNPs contain elF2C2 (Argonaute 2), DDX20, GEMIN4 and microRNA. Animal microRNAs target in particular developmental genes [7]. In contrast, genes involved in functions common to all cells, such as gene expression, has resulted in the production of microarrays dubbed MMChips containing all then known miRNAs for human, mouse, rat, dog, C. elegans and Drosophila [8]. All the miRNAs in plants are derived from the sequential DCL1 cleavages from pri-miRNA to give pre-miRNA (or miRNA precursor). But the mirtrons bypass the DCL1 cleavage and enter as pre-miRNA in the miRNA maturation pathway. Mirtrons are the type of microRNAs which are located in the introns of the mRNA encoding host genes. Many miRNAs are evolutionarily conserved. Although the biological functions of most miRNAs are unknown, miRNAs are predicted to regulate up to $30 \%$ of the genes within the human genome.

\section{Formation and Processing of miRNA}

The genes encoding miRNAs are much longer than the processed mature miRNA molecule; miRNAs are first transcribed as primary transcripts or pri-miRNA with a cap and poly-A tail and processed to short, 70-nucleotide stemloop structures known as pre-miRNA in the cell nucleus. This processing is performed in animals by a protein complex known as the Microprocessor complex, consisting of the nuclease Drosha and the double-stranded RNA binding protein Pasha [9]. These pre-miRNAs are then processed to mature miRNAs in the cytoplasm by interaction with the endonuclease Dicer, which also initiates the formation of the RNA-induced silencing complex (RISC) [10].This complex is responsible for the gene silencing observed due to miRNA expression and RNA interference. The pathway in plants varies slightly due to their lack of Drosha homologs; instead, Dicer homologs alone effect several processing steps [11]. The pathway is also different for miRNAs derived from intronic stem-loops; these are processed by Dicer but not by Drosha [12]. Either the sense strand or antisense strand of 
DNA can function as templates to give rise to miRNA [13]. Efficient processing of pri-miRNA by Drosha requires the presence of extended singlestranded RNA on both 3'- and 5'-ends of hairpin molecule [14]. These ssRNA motifs could be of different composition while their length is of high importance if processing is to take place at all. A bioinformatics analysis of human and fly primiRNAs revealed very similar structural regions, called 'basal segments', 'lower stems', 'upper stems' and 'terminal loops'; based on these conserved structures, thermodynamic profiles of pri-miRNA have been determined [15]. The Drosha complex cleaves the RNA molecule $~ 22$ nucleotides away from the terminal loop [16]. Most pre-miRNAs don't have a perfect doublestranded RNA (dsRNA) structure topped by a terminal loop. There are few possible explanations for such selectivity. One could be that dsRNAs longer than 21 base pairs activate interferon response and anti-viral machinery in the cell. Another plausible explanation could be that the thermodynamic profile of pre-miRNA determines which strand will be incorporated into Dicer complex. Indeed, clear similarities between pri-miRNAs encoded in respective (5'- or 3'-) strands have been demonstrated [15]. When dicer cleaves the pre-miRNA stem-loop, two complementary short RNA molecules are formed, but only one is integrated into the RISC complex. This strand is known as the guide strand and is selected by the argonaute protein, the catalytically active RNase in the RISC complex, on the basis of the stability of the $5^{\prime}$ end [17]. The remaining strand, known as the miRNA, antiguide or passenger strand, is degraded as a RISC complex substrate [18]. After integration into the active RISC complex, miRNAs base pair with their complementary mRNA molecules and inhibit translation or sometimes induce mRNA degradation by argonaute proteins, the catalytically active members of the RISC complex [19] It is as yet unclear how the activated RISC complex locates the mRNA targets in the cell, though it has been shown that the process is not coupled to ongoing protein translation from the mRNA [20].

\section{Expression of micro RNA}

Measuring the quantity of miRNAs in tissues of different physiological and pathological conditions is an important first step to investigate the functions of miRNAs' [29]. Small-RNAs that do not encode proteins themselves can regulate messenger RNA molecules. Cohen and colleagues are credited with the discovery of one miRNA gene, bantam, in a nonprotein-coding region of the fruit fly genome. They found that bantam encodes a sequence that stimulates cell proliferation and prevents apoptosis. Cohen and his colleagues at the Heidelberg-based EMBL took a bioinformatics approach to identify additional targets in the fruit fly Drosophila melanogaster, a commonly-used model for genetic research. But, in mammals there are often multiple versions of the same miRNA genes which makes them difficult to study by using knockout (gene deletion) approaches, and conventional in situ hybridisation experiments are unreliable as miRNAs are too short to generate a signal [21]. Following identification, miRNAs have rapidly taken the center stage as key regulators of gene expression. As current knowledge of the miRNA biosynthetic pathway and its protein components, as well as the processes it regulates via miRNAs, which are known to exert a variety of biological functions in eukaryotes. Although the relative importance of miRNAs remains to be fully appreciated, deregulated protein expression resulting from either dysfunctional miRNA biogenesis or abnormal miRNA-based gene regulation may represent a key etiologic factor in several, as yet unidentified, diseases. Hence, it is need to better understand the complexity of the basic mechanisms underlying miRNA biogenesis and function [22]. They negatively regulate gene expression through translational inhibition or mRNA cleavage expression of specific miRNAs contributes to human disease has fueled much interest in profiling the expression of these molecules. Realtime quantitative PCR (RQ-PCR)is a sensitive and reproducible gene expression quantitation technique which is now being used to profile miRNA expression in cells and tissues. The expression of five miRNA genes (let-7a, miR-10b, miR-16, miR-21 and miR-26b) and three small nucleolar RNA genes (RNU19, RNU48 and Z30) was examined across malignant, benign and normal breast tissues to determine the most appropriate normalization strategy[23].Real-time PCR is also effective for quan-tifying the expression of plant miRNAs miRNAs, was polyadenylated and reverse-transcribed with a poly $(\mathrm{T})$ adapter into cDNAs for real-time PCR using the miRNA-specific forward primer and the sequence complementary to the poly $(\mathrm{T})$ adapter as the reverse primer. Several Arabidopsis miRNA sequences were tested using SYBR® Green reagent, demonstrating that this method, using as little as $100 \mathrm{pg}$ total RNA, could readily discriminate the expression of miRNAs having as few as one nucleotide sequence difference. This method also revealed miRNA tissue-specific expression patterns that cannot be resolved by Northern blot analysis and may therefore be widely useful for characterizing miRNA ex $\neg$ pression in plants as well as in animals [24]. An unbiased assessment of the relative importance of miRNA-based regulation in the human genome consistent with recent independent studies and overall, $\sim 45 \%$ of the 108 highly conserved motifs in 3'-UTRs appear to be related to miRNA regulation and $\sim 5000$ human 
genes ( $20 \%$ of the genome) are likely to be regulated by miRNAs throu0gh these conserved motifs[25]. Recent work by researchers has revealed the existence of a class of small noncoding RNA species, which have critical functions across various biological processes. They use a new, bead-based flow cytometric miRNA expression profiling method to present a systematic expression analysis of 217 mammalian miRNAs from 334 samples, including multiple human cancers. The miRNA profiles are surprisingly informative, reflecting the developmental lineage and differentiation state of the tumours. They were able to successfully classify poorly differentiated tumours using miRNA expression profiles, whereas messenger RNA profiles were highly inaccurate when applied to the same samples. These findings highlight the potential of miRNA profiling in cancer diagnosis [26]. Researchers have established a MIR-specific oligonucleotide microarray system that enables efficient analysis of the expression of the human miRNA. Expression profiling of 150 miRNA in five human tissues and in HeLa cells revealed a good overall concordance present novel data on miRNA expression in thymus, testes, and placenta, and have identified miRNA highly enriched in these tissues. Taken together, these results highlight the increased sensitivity of the DNA microarray over other methods for the detection and study of miRNA and the immense potential in applying such microarrays for the study of MIRs in health and disease [27]. Microarray technology is frequently applied to monitor miRNA expression levels but is challenged by (i) the short length of miRNAs that offers little sequence for appending detection molecules; (ii) low copy number of some miRNA; and (iii) a wide range of predicted melting temperatures (Tm) versus their DNA complementary sequences [31]. Using microarrey to determine the microRNA expression profile during mouse brain development and observed a temporal wave of gene expression of sequential classes of microRNAs [28]. Some miRNAs are expressed in a cell- or tissue-specific manner and may contribute to the establishment and maintenance of cellular identity. Recent studies indicate that tissue-specific miRNAs may function at multiple hierarchical levels of gene regulatory networks, from targeting hundreds of effector genes incompatible with the differentiated state to controlling the levels of global regulators of transcription and alternative pre-mRNA splicing. This multilevel regulation may allow individual with immunophenotype, survival and transformation from follicular lymphoma [30].

\section{Precursors in synaptic fractions of adult mouse forebrain}

The brain expresses a wide variety of miRNAs, some of which show regional and cell type specificity [32, 33]. miRNAs are also expressed in dendrites where they regulate local protein translation [34]. New neurons are generated in the adult brain from stem cells found in the subventricular zone (SVZ). These cells proliferate in the SVZ, generating neuroblasts which then migrate to the main olfactory bulb (MOB), ending their migration in the glomerular layer (GLL) and the granule cell layer (GCL) of the MOB [27]. A recent experimental study of adult mouse forebrain reported the expression of miRNAs in synaptoneurosomes (SYN), a synaptic fraction that is enriched in pinched-off dendritic spines [35]. Within mouse forebrain, a subset of microRNAs is significantly enriched in synaptoneurosomes and a subset are significantly depleted relative to total forebrain homogenate. In case of the pre-miRNA hairpin precursors of synaptically enriched microRNAs exhibit significantly different structural features than those that are non-enriched or depleted. Precursors of synaptically enriched microRNAs tend to have a) shorter uninterrupted doublestranded stem segments, and b) more symmetrical bulges containing a single nucleotide on each side. These structural differences may provide a basis for the differential binding of proteins that mediate dendritic transport of premiRs, or that prevent pre-miRs from being prematurely processed into mature miRNAs during the transport process [36]. The neurotrophin brain-derived neurotrophic factor plays an important role in the activity-dependent regulation of synaptic structure and function, particularly of the glutamatergic synapses. Recent advances in the understanding of the regulation of the glutamatergic synapse by BDNF and its implications in synaptic plasticity [37]. The presence of AMPA receptor subunits and the possible dynamic control of their surface exposure at the presynaptic membrane; they demonstrate that the AMPA receptor subunits GluR1 and GluR2 are expressed and organized in functional receptors in axonal growth cones of hippocampal neurons. At steady state, major pools of AMPA receptor subunits reside in synaptic vesicle membranes and can be recruited to the presynaptic membrane as functional receptors in response to depolarization [38]. Paralemmin (Palm) is a prenyl-palmitoyl anchored membrane protein that can drive membrane and process formation in neurons. Earlier studies have shown brain preferred Palm expression, and the Palm gene may be regulated by Pax6. The expression profile of Palm protein in the embryonic, newborn and adult mouse eye as well as dissociated retinal neurons was determined by confocal immunofluorescence 
[39]. In adult male rat hippocampal formation, significant localization was demonstrated for both cytochromes P45017 $\alpha$ and P450 aromatase, in pyramidal neurons in the CA1-CA3 regions, as well as in the granule cells in the dentate gyrus, by means of immunohistochemical staining of slices. Finding of a P450scc-containing neuronal system for pregnenolone synthesis, these imply that $17 \beta$-estradiol is synthesized by $P 45017 \alpha$ and P450 aromatase localized in hippocampal neurons from endogenous cholesterol. This synthesis may be regulated by a glutamatemediated synaptic communication that evokes Ca2+ signals [48]. Also, Plasma membrane $\mathrm{Ca} 2+-A T P a s e s$ (PMCAs) have high affinity $\mathrm{Ca} 2+$ transporters actively involved in intracellular $\mathrm{Ca} 2+$ homeostasis. Researchers have seen that upregulation of PMCA activity and PMCA isoforms expression during brain development in mouse, with specific localizations mainly in cerebellum. Overall, findings support a close relationship between the ontogeny of PMCA isoforms and specific requirements of $\mathrm{Ca} 2+$ during development of different brain areas.[40].In adult mice that destruction of the dopaminergic neurons in the substantia nigra and ventral tegmental area in a 6-hydroxydopamine model of Parkinson's disease reduced the number of proliferating neural precursors in the subventricular zone of the anterior lateral ventricle by $40 \%$. The effect on neural precursor proliferation correlated with the extent of dopaminergic denervation in the neighboring neostriatum. This identifies dopamine as one of the few known endogenous regulators of adult neurogenesis with implications for the potential use of endogenous neural precursors in cell replacement strategies for Parkinson's disease [50]. Dopamine receptors are also associated with a mammalian central nervous system, dopamine receptors are coupled to G-proteins. Typically, dopamine receptor activation leads to changes in intracellular cAMP levels [41, 42] and triggers a signaling cascade that culminates in gene transcription. Based on G-proteinpartners and intracellular signaling mechanisms, two classes of dopamine receptors are recognized [43]. The D1-like receptors are Gs/olf coupled and their activation results in increased intracellular cAMP. The D2-like receptors are $\mathrm{Gi} / \mathrm{o}$ coupled and their activation decreases intracellular cAMP [44]. In order to achieve temporally controlled somatic mutagenesis, the Cre/loxP-recombination system has been complemented with the tamoxifen-inducible fusion protein consisting of Cre recombinase and the mutated ligand binding domain of the human estrogen receptor (CreERT2). To induce recombination of conditional alleles in neurons of the adult forebrain, they generated a bacterial artificial chromosome-derived transgene expressing the CreERT2 fusion protein under control of the regulatory elements of the CaMKIla gene [45]. In the developing mouse brain, the highest $\mathrm{Bcl}-\mathrm{XL}$ expression is seen at the peak of neurogenesis, whereas the peak of Bax expression coincides with the astrogenic period. Such observations suggest an active role of the $\mathrm{Bcl}-2$ family proteins in the generation of neurons and astrocytes. Using combinations of gain- and loss-of-function assays in vivo and in vitro, we provide evidence for instructive roles of these proteins in neuronal and astrocytic fate specification. Specifically, in Bax knockout mice, astrocyte formation was decreased in the developing cortices. Overexpression of Bcl-XL and Bax in embryonic cortical precursors induced neural and astrocytic differentiation, respectively. Importantly, inhibition of caspase activity, dimerization, or mitochondrial localization of Bcl$\mathrm{XL} / \mathrm{Bax}$ proteins indicated that the differentiation effects of Bcl-XL/Bax are separable from their roles in cell survival and apoptosis. Lastly, Activation of intracellular signaling pathways and expression of basic helix-loop-helix transcriptional factors specific for the Bcl-2 protein-mediated differentiation [46].Another precursor of Abl-interactor (Abi) family of adaptor proteins has been linked to signaling pathways involving the Abl tyrosine kinases and the Rac GTPase. Loss of Abi2 also resulted in cell migration defects in the neocortex and hippocampus, abnormal dendritic spine morphology and density, and severe deficits in short- and long-term memory. These findings support a role for $\mathrm{Abi} 2$ in the regulation of cytoskeletal dynamics at adherens junctions and dendritic spines, which is critical for intercellular connectivity, cell morphogenesis [49]. Loss of presenilin function in adult mouse brains causes memory loss and age-related neurodegeneration. Since presenilin possesses-secretase-dependent and independent activities. Generated postnatal forebrain-specific nicastrin conditional knock-out (cKO) mice, in which nicastrin, a subunit ofsecretase, is inactivated selectively in mature excitatory neurons of the cerebral cortex. Nicastrin cKO mice display progressive impairment in learning and memory and exhibit age-dependent cortical neuronal loss, accompanied by astrocytosis, microgliosis, and hyperphosphorylation of the microtubuleassociated protein Tau [51].

\section{Micro RNA induced during Adipogenesis}

Adipogenesis is a highly regulated process in which, coordinated cascade of transcription factors leads to the formation of mature adipocytes [52]. This cascade begins with the transient expression of CCAAT/enhancer binding protein $\beta(\mathrm{C} / \mathrm{EBP} \beta)$ and $\mathrm{C} / \mathrm{EBP} \delta$ which activate $\mathrm{C} / \mathrm{EBPa}$ and peroxisome proliferator-activated receptor $\mathrm{Y}$ (PPARY).C/EBPa and PPARy together coordinate the expression ofadipogenic genes 
underlying the phenotype of terminally differentiated adipocytes. This terminal differentiation is characterized by the induction of genes including glycerol-3-phosphate dehydrogenase (GPDH), hormone-sensitive lipase (HSL), fatty acid synthase (FASN), fatty acid binding proteins (FABPs), perilipin (PLIN), and the production and secretion of adipokines such as leptin (LEP), adiponectin (ADIPOQ), adipsin (CFD), tumor necrosisfactor alpha (TNFa), visfatin (NAMPT) and retinol binding protein 4 (RBP4). Additional transcription factors, such as sterol-regulatory element binding transcription factor 1 (ADD1/SREBP1) can further modulate this terminal differentiation process [53]. Researchers performed a miRNA binding site analysis to identify miRNAs potentially targeting genes regulated during adipocyte or osteoblast differentiation and significantly overrepresented miRNA targets in the eight distinct gene clusters. Out of the 1606 genes identified as being differentially expressed during differentiation the 3'-UTR.sequence could be obtained for 1147 of them. All of these had at least one exact antisense match with the 7-nt seed(base 2-8 at the 5' end) from the 470 human miRNA sequences The distribution of statistically enriched 3'-UTR miRNA motifs varied across the clusters, with genes in cluster 1having no detectable motifs and genes in cluster 5 havingthe most miRNA motif with a unique 3'UTR that were differentially expressed during adipocyte and osteoblast differentiation are potential targets for 30 miRNAs with significantly over-represented motifs [54]. Tumour necrosis factor, a proinflammatory cytokine, is a potent negative regulator of adipocyte differentiation. However, mechanism of TNF-mediated antiadipogenesis remains incompletely understood. TNF-inhibits adipogenesis of 3T3-L1 preadipocytes by preventing the early induction of the adipogeni transcription factors peroxisome proliferator-activated receptor-and CCAAT/enhancer binding protein [55]. Peroxisome proliferator-activated receptors- $y$ (PPARy) plays a critical role in glucose and lipid metabolism, macrophage function, and adipogenesis. It is a nuclear hormone receptor, activated through ligand binding, which results in allosteric changes in receptor conformation, recruitment of coactivators, assembly of a transcriptional complex, there regulates gene expression. Thiazolidinedione (TZD) is one of the agonist of PPARy receptor which has been a medication for diabetic mellitus for years. Treatment with TZDs leads to selective accumulation of subcutaneous adipose tissue.adipogenesis induction in D1 cells is initiated by activation of peroxisome proliferatoractivated receptor-y. The results revealed that pioglitazone induces adipogenesis in D1 cells in dose dependent manner and decreases alkaline phosphatase activity in D1 cells. Interestingly, adipogenesis was not blocked by bisphenol A diglycidyl ether, a peroxisome proliferatoractivated receptor- $y$ antagonist. A peroxisome proliferator-activated receptor- $\gamma$-mediated reporter gene assay showed no response to pioglitazone.Dexamethasone-induced

adipogenesis can be repressed by mifepristone (RU486), an antagonist of glucocorticoid receptor. The results disclosed that mifepristone cannot counteract dexamethasone-induced adipogenesis, and mifepristone itself induced adipogenesis in D1 cells. Moreover, glucocorticoid receptor-mediated reporter gene assay was not responsive to dexamethasone or mifepristone.researchers concluded that the adipogenesis induced by pioglitazone and dexamethasone in D1 cells may not occur via a peroxisome proliferator-activated receptor- $y$ and glucocorticoid receptor pathway. Results suggested that the adipogenesis induced by glucocorticoids and pioglitazone is directed by a multiple cell signaling pathway. Finally, data from microarray analysis confirmed this adipogenesis pathway, as several adipogenesis-related genes are provoked by DEX [56]. Although miRNAs may be involved in modulating adipocyte function, mild down-modulations of the upregulated miRNAs do not appear to affect 3T3-L1 pre-adipocyte differentiation [57]. miRNAs were differentially regulated during in vitro and in vivo adipogenesis. Importantly, miRNAs that were induced during adipogenesis were downregulated in adipocytes from both types of obese mice and vice versa. These changes are likely associated with the chronic inflammatory environment, since they were mimicked by TNF- $\alpha$ treatment of differentiated adipocytes. Ectopic expression of miR-103 or miR-143 in preadipocytes accelerated adipogenesis, as measured both by the upregulation of many adipogenesis markers and by an increase in triglyceride accumulation at an early stage of adipogenesis [58]. They further assessed the contribution of the chronic inflammatory environment in obese adipose tissue to the dysregulated miRNA expression by tumor necrosis factor (TNF)- $\alpha$ treatment of adipocytes [59]. Researchers showed that down-regulation of two over-expressed miRNAs, miR-27a and 27b allowed culture-activated rat HSCs to switch to a more quiescent HSC phenotype, with restored cytoplasmic lipid droplets and decreased cell proliferation. Mechanistically, retinoid $\mathrm{X}$ receptor a was confirmed to be the target of miR$27 \mathrm{a}$ and $27 \mathrm{~b}$. These results indicated a new role and mechanism of miR-27a and $27 \mathrm{~b}$ in regulating fat metabolism and cell proliferation during HSCs activation [60]. 


\section{Cells are down regulated in obesity}

Obesity, the excess deposition of adipose tissue, is among the most pressing health problems both in the Western world and in developing countries, growth of adipose tissue is the result of the development of new fat cells from precursor cells. Researchers investigated the role of miRNAs in the regulation of adipogenic differentiation. They demonstrate that the miR-27 gene family is downregulated during adipogenic differentiation. Overexpression of miR-27 specifically inhibited adipocyte formation, without affecting myogenic differentiation. We also found that expression of miR-27 resulted in blockade of expression of PPAR gamma and C/EBPalpha, the two master regulators of adipogenesis. Importantly, expression of miR-27 was increased in fat tissue of obese mice and was regulated by hypoxia, an important extracellular stress associated with obesity. data strongly suggest that miR-27 represents a new class of adipogenic inhibitors and may play a role in the pathological development of obesity [61].This study opens new perspectives in understanding the biology of human WAT and its pathologic changes indicative of tissue deterioration associated with the development of obesity $[62,65]$. Type 2 diabetes is associated with excessive food intake and a sedentary lifestyle. Local inflammation of white adipose tissue induces cytokine-mediated insulin resistance of adipocytes, results in enhanced lipolysis within these cells. The fatty acids that are released into the cytosol can be removed by mitochondrial $\beta$-oxidation. Elevated fatty acid concentrations in the cytosol of adipocytes induce mitochondrial uncoupling and thereby allow mitochondria to remove much larger amounts of fatty acids. The number and activity of mitochondria within adipocytes contribute to the threshold at which fatty acids are released into the circulation, leading to insulin resistance and type 2 diabetes [63]. In mammalian cells, mTOR is part of two different kinase complexes: mTORC1 composed of mTOR, raptor and mLST8, and mTORC2 containing mTOR. Researchers showed that mTORC2 itself is a potent regulator of mammalian cell size and cell cycle via a mechanism involving the Akt/TSC2/Rheb cascade. This data is important for the understanding of the molecular development of the many human diseases caused by deregulation of upstream and downstream effectors of mTOR [64].The observations have led to the formation of an exciting concept, adipose tissue hypoxia (ATH), in the understanding of major disorders associated with obesity. ATH may provide cellular mechanisms for chronic inflammation, macrophage infiltration, adiponectin reduction, leptin elevation, adipocyte death, endoplasmic reticulum stress and mitochondrial dysfunction in white adipose tissue in obesity .ATH may represent a unified cellular mechanism for a variety of metabolic disorders and insulin resistance in patients with metabolic syndrome [66]. Plasminogen activator inhibitor-1 (PAl-1) is a cytokine upregulated in obesity whose expression is correlated with a poor prognosis in breast cancer. As a key mediator of adipogenesis and regulator of adipokine production, peroxisome proliferator-activated receptor-y (PPAR-y) is involved in PAI-1 expression from adipose tissue.current knowledge linking PPAR-y and PAl-1 expression to high-fat diet and obesity in the risk of breast cancer [67]. Interestingly, SOX6 was previously found to be essential to both cartilage formation/chondrogenesis and obesity-related insulin resistance, suggesting the gene's dual role in both bone and fat.[68].researchers show that $\mathrm{HSP} 72+/+$ mice display greater mitochondrial enzyme activity in the liver, adipose tissue and skeletal muscle, corresponding to reduced plasma free fatty acid levels, white adipose tissue mass and alterations in circulating adipokines. These data identify HSP72 as being pivotal in protecting against obesity-induced insulin resistance possibly by blocking JNK and/or by up-regulation of mitochondrial oxidative capacity [69]. Genes regulating the extracellular matrix and cell death showed a strong downregulation after long-term weight reduction. This likely reflects a new stable state at the molecular level in AT. Further studies are warranted to elucidate the mechanisms of these genetic factors [70].

\section{Micro RNA functions in human embryonic stem cells}

Human embryonic stem (hES) cells have unique abilities to divide indefinitely without differentiating and potential to differentiate into more than 200 cell types. These properties make hES cells an ideal model system for understanding early human development and for regenerative medicine. Molecular mechanisms including cellular signaling and transcriptional regulation play important roles in $\mathrm{hES}$ cell differentiation. In hES cells we found that 276 miRNAs were expressed; of these, a set of 30 miRNAs had significantly changed expression during differentiation. Findings reveal the evolutionary emergence, of miRNAs involved in regulating early human development [71]. Patterns of expression in the progression from hESC to differentiated cells that suggest a role for selected miRNAs in maintenance of the undifferentiated, pluripotent state; profiling of the hESC "miRNA-ome" provides an insight into molecules that control cellular differentiation and maintenance of the pluripotent state, findings that have broad implications in development, homeostasis and human disease states [72]. overexpression of endoderm-specific 
miRNA(miRNA122) may affect hESC differentiation [73]. Researchers showed that muscle-specific miRNAs reinforce the silencing of non muscle genes during cell lineage commitment and suggest that miRNAs may have general utility in regulating cell fate decisions from pluripotent ES cells [74]. Report on identification of human lin-28 mRNA as a regulatory target of human miR-125b and its homolog miR-125a. Studies of miR-125b function in mouse P19 embryonal carcinoma cells induced to develop into neurons suggest a role for this regulatory miRNA in mammalian neuronal differentiation, since its increased concentration in these cells contributes to lin-28 downregulation. Gene repression by other miRNAs that are imperfectly complementary to their targets, lin-28 downregulation by miR-125 involves reductions in both translational efficiency and mRNA abundance [75]. Understanding the biology of human embryonic stem cells, and how cells can be controlled to differentiate into desirable neuronal cells once implanted into human central nervous system .fundamental knowledges are essential for the future development of stem cell therapies for a wide range of mental disorders and age-dependent neurodegenerative diseases [76].The mir-302 microRNA family (mir-302s) is expressed most abundantly in slow-growing human embryonic stem (ES) cells, and quickly decreases after cell differentiation and proliferation. The mir-302 transfected cells, namely, miRNA-induced pluripotent stem (mirPS) cells, not only expressed many key ES cell markers, such as Oct3/4, SSEA-3, SSEA-4 ,Sox2, and Nanog, but also had a highly demethylated genome similar to a reprogrammed zygotic genome mir-302s not only function to reprogram cancer cells into an ES-like pluripotent state but also to maintain this state under a feeder-free cultural condition, which may offer a great opportunity for therapeutic intervention [77]. miRNA genes in hES cells were expressed from Chromosomes 19 and X [78] and demonstrated the significant upregulation of miR520 cluster in hES cells [79]. LIN-28 is a highly specific embryonic stem cell marker and is one of four "stemness" factors used to reprogram adult fibroblasts into induced pluripotent stem cells [80]. Signature miRNAs, such as the miR-302 family, the miR-200 family have been reported in human [81] and mouse embryonic stem cells [82].

\section{Micro RNA biogenesis in embryonic stem cells and cancer}

miRNA biogenesis includes miRNA transcription in the nucleus, the export of miRNAs from the nucleus to the cytoplasm, and subsequent processing and maturation in the cytoplasm.In most cases, the transcription of miRNA genes is mediated by RNA polymerase II (Pol II), resulting in long primary miRNA (pri-miRNA) transcripts with a fold-back structure comprising a stem loop along with flanking segments [83]. A few recent reports have shown the involvement of RNA polymerase III (Pol III) in miRNA transcription [84].The sequence of the miRNA remains embedded in the arms of the stem loop. The primiRNA contains the 7-methylguanosine cap and a poly $(A)$ tail, which is unique for Pol II transcripts, similar to mRNAs. However, the cap and poly $(A)$ tail are removed during miRNA processing. miRNA promoters have been identified in many studies and reported to have typical Pol II elements such as a TATA box, although the recent report suggests that members of the human chromosome19 miRNA cluster (miR-5151, miR-517a, miR-517c and miR-519a-1) are interspersed among Alu repeats and expressed through Pol III.all animal miRNAs studied so far are usually not exactly complementary to their mRNA targets, and seem to inhibit protein synthesis while retaining the stability of the mRNA target (Ambros 2004). It has been suggested that transcripts may be regulated by multiple miRNAs and an individual miRNA may target numerous transcripts.[85].miRNAs act on cellular transcripts by two different mechanisms. The first mechanism is cleavagedependent RNA degradation of the transcript at the site where the miRNA binds. The other mechanism is miRNA-mediated translational repression of the target transcript, while the miRNA binds to the target 3'-UTR with several mismatches. This partial complementarity allows for miRNAs targeting multiple 3'-UTRs [86]. A key question that arises from the targeting of multiple transcripts is whether there has been evolutionary enrichment in targets that are involved in similar cellular functions. microRNA biogenesis and activity, with particular focus on mechanisms of post-transcriptional control. Further understanding of the regulation of microRNA biogenesis and activity will undoubtedly provide important insights into normal development as well as pathological conditions such as cardiovascular disease and cancer [87]. Recent work in embryonic stem (ES) cells has demonstrated that core features of the network can be discovered by focusing on key transcriptional regulators and their target genes [88]. The present invention relates to a method and composition for generating a non-naturally occurring intron and its intronic components capable of being processed into mir-302-like RNA molecules in mammalian cells and thus inducing certain specific gene silencing effects on differentiation-related and fate-determinant genes of the cells, resulting in reprogramming the cells into a pluripotent embryonic stem (ES)-cell-like state. The ES-like cells so obtained are strongly express hES cell markers, such as Oct3/4, SSEA-3 and SSEA-4, and can be guided into 
various tissue cell types by treating certain hormones and/or growth factors under a feederfree cell culture condition in vitro, which may be used for transplantation and gene therapies[89].Aberrant expression of many miRNAs is linked to cancer and other diseases. Expression patterns of miRNAs correlate with tissue of origin [90] prognosis and with clinical cancer behaviors making miRNAs valuable tissue-based biomarkers [91]. miRNAs known to be expressed in epithelial ovarian cancer and provides a useful resource for future studies of the role of miRNAs in the pathogenesis and early detection of ovarian cancer [92]. micro-RNA could mediate potent and specific gene silencing has raised high hopes for cancer therapeutics. Three strategies are currently being developed: 1) De novo RNAi programming using synthetic miRNA to target the expression of genes. 2) Strengthening or recapitulation of the physiologic targeting of messenger RNA by specific miRNA. 3) Sequence-specific inhibition of mi RNA functions by nucleic acid analogs [93].

\section{References}

[1] Lee R.C., Feinbaum R.L., Ambros V. (1993) Cell 75 (5): 843-54.

[2] He S., Su H., Liu C., et al. (2008) BMC Genomics 9: 236.

[3] Kandhavelu M., Lammi C., Buccioni M., Dal Ben D., Volpini R., Marucci G. (2009) Journal of Bioinformatics and Sequence Analysis 1 (2): 031-040.

[4] Eulalio A., Huntzinger E., Nishihara T., Rehwinkel J., Fauser M., Izaurralde E. (2009) RNA 15 (1): 21-32.

[5] Tan Y., Zhang B., Wu T., et al. (2009) BMC Mol. Biol. 10 (1):

[6] Hawkins P.G., Morris K.V. (2008) Cell Cycle 7 (5): 602-7.

[7] Mourelatos Z., Dostie J., Paushkin S., et al. (2002) Genes Dev. 16 (6): 720-8.

[8] Jaclyn, Shingara;(2005) RNA 11: 14611470.

[9] Denli A.M., Tops B.B., Plasterk R.H., Ketting R.F., Hannon G.J. (2004) Nature 432 (7014): 231-5.

[10] Bernstein E., Caudy A.A., Hammond S.M., Hannon G.J.(2001) Nature 409 (6818): 363-6.

[11] Kurihara Y., Watanabe Y. (2004) Proc. Natl. Acad. Sci. U.S.A. 101 (34): 12753-8.

[12] Gao F.B. (2008) Trends Neurosci. 31 (1): 20-6.

[13] Stark A., Bushati N., Jan C.H., et al. (2008) Genes Dev. 22 (1): 8-13.

[14] Zeng Y., Cullen B.R. (2005) J. Biol. Chem. 280 (30): 27595-603.

[15] Han J., Lee Y., Yeom K.H., Nam J.W., Heo I., Rhee J.K., Sohn S.Y., Cho Y., Zhang B.T., Kim V.N. (2006) Cell 125 (5): 887901.
[16] Zeng Y., Yi R., Cullen B.R. (2005) Embo J. 24 (1): 138-48.

[17] Preall J.B., He Z., Gorra J.M., Sontheimer E.J. (2006) Curr. Biol. 16 (5): 530-5.

[18] Gregory R.I., Chendrimada T.P., Cooch N., Shiekhattar R. (2005) Cell 123 (4): 63140.

[19] Liu J., Carmell M.A., Rivas F.V., Marsden C.G., Thomson J.M., Song J.J., Hammond S.M., Joshua-Tor L., Hannon G.J. (2004) Science 305:1437-1441..

[20] Sen G.L., Wehrman T.S., Blau H.M. (2005) Differentiation 73 (6): 287-93.

[21] Ouellet D.L., Perron M. P., Lise-Andrée Gobeil, Pierre Plante and Patrick Provost (2006) J Biomed Biotechnol., 69616, PMC1559927.

[22] Meister G., Tuschl T. (2004) Nature, 431:343-349.

[23] Davoren P.A., McNeill R.E., Lowery A.J., Kerin M.J., Miller N. ((2008) BMC Mol Biol., 9: 76

[24] Shi R., Chiang V.L. (2005) BioTechniques, 39:519-525.

[25] Xie X., Lander E.S., Kellis M. (2005) Broad Institute of MIT and Harvard, Cambridge MA 02139; Whitehead Institute for Biomedical Research, Cambridge MA 02139.

[26] Lu J., Getz G., Miska E.A., Alvarez S. E., Lamb J., Peck D., Mak B.L., Ferra R.H. (2005) Nature, 435(7043):834-8

[27] Barad O., Eti Meiri Amir Avniel, Ranit Aharonov, Adi Barzilai, Research Triangle Park, North Carolina 27709, USA.

[28] Miska E.A., Ezequiel Alvarez-Saavedra, Matthew T. (2007) Verh Dtsch Ges Pathol 91: 214-20.

[29] Liang Y., Ridzon D., Wong L., Caifu C. (2007) BMC Genomics, 8: 166, PMC1904203.

[30] Lawrie Charles H., Chi Jianxiang, Taylor Stephen, Tramonti Daniela, Ballabio Erica, Palazzo Stefano, Saunders Nigel J., Pezzella Francesco, Boultwood Jacqueline, Wainscoat James S., Hatton Christian S. R. (2009) Journal of cellular and molecular medicine 13(7):1248-60..

[31] Mirco C., Sabine S., Vladimir B., Hentze M.W., Muckenthaler M.U. (2008) Nature protocols, 3(2):321-9.

[32] Quintana M.L., Rauhut R., Yalcin A., Meyer J., Lendeckel W., Tuschl T. (2002) Curr Biol., 12:735-739.

[33] Kosik K.S. (2006) Nat Rev Neurosci., 7:911-920.

[34] Schratt G.M., Tuebing F., Nigh E.A., Kane C.G., Sabatini M.E., Kiebler M., Greenberg M.E. (2006) Nature, 439:283-289. 
[35] Lugli G., Torvik V.I., Larson J., Smalheiser N.R. (2008) Neurochem.;106:650-661.

[36] Smalheiser N.R. (2008) Biol Direct., 3: 44. PMCID: PMC2588566

[37] Carvalho A. L., Caldeira M. V., Santos S. D. and Duarte C.B. (2008) Br J Pharmacol. 153(S1): S310-S324.

[38] Schenk U., Verderio C., Benfenati F., Matteoli M. (2003) EMBO J., 22(3): 558-568.

[39] Castellini M., Wolf L.V., Chauhan B.K., Galileo D.S., Kilimann M.W., Cvekl A., Duncan M. K. (2005) BMC Ophthalmol, 5: 14.

[40] Marcos D.M., Sepulveda R., Berrocal M. and Mata A.M. (2009) BMC Neurosci.; PMC2749858

[41] Monsma F.J., Mahan L.C., McVittie L.D., Gerfen C.R., Sibley D.R. (1990) Proc Natl Acad Sci USA;87:6723-6727.

[42] Robinson S.W., Caron M.G. (1996) J Neurochem., 67:212-219.

[43] Lévesque M., Gagnon S., Parent A., Deschênes M. (1996) Cerebral Cortex 6:759-770.

[44] Araki K.Y., Sims J.R., Bhide P.G. (2007) Brain Res., 1156: 31-45.

[45] Erdmann G., Schütz G., Berger S. (2007) BMC Neurosci., 8: 63.

[46] Chang M.Y., Sun W., Ochiai W. (2007) Mol Cell Biol.; 27(12): 4293-4305.

[47] Bagley J., LaRocca G., Jimenez J.A., and Nathaniel N. Urban (2007) BMC Neurosci., 8: 92.

[48] Hojo Y., Taka-aki Hattori (2004) Proc Natl Acad Sci U S A., 101(3): 865-870.

[49] Matthew G., Galina D. (2004) Mol Cell Biol., 24(24), 10905-10922.

[50] Baker S.A. , Baker K. A. and Theo Hagg (2005) BioTechnique-s 39:519-525.

[51] Katsuhiko T.,Guiquan C. (2009) Neurosci.;29(22):7290-301.

[52] Rosen E.D. (2005) Prostaglandins Leukot Essent Fatty Acids 73:31-34.

[53] Sethi J.K., Vidal P. A. (2007) J Lipid Res, 48:1253-1262.

[54] Scheideler M., Sanchez F. (2008) BMC Genomics, 9:340.

[55] Cawthorn W. P. (2007) Cell Death Differ. 14(7):1361-73.

[56] Hung Shao-Hung, Lu Yen-Mou, Huang Hsuan-Ti, Lin Yen-Ko, Chang Je-Ken, Chen Jian-Chih, Tien Yin-Chun, Huang Peng-Ju, Chen Chung-Hwan, Liu PingCheng, Chao David. (2007) Knee surgery, sports traumatology, arthroscopy: official journal of the ESSKA, 15(5):578-86.

[57] Kazuaki Kajimoto, Hiroaki Naraba, Naoharu Iwai (2006) RNA12 (9), 1626-32.

[58] Xie H., Lim B., Lodish H.F. (2009) Diabetes.;58(5):1050-7.
[59] Xie H.B., Lodish H.F. (2009) Diabetes 58(5) 1050-7.

[60] Ji J., Zhang J., Huang G., Qian J., Wang X., Mei S. (2009) FEBS Lett. 583(4):759-66.

[61] Lin Q., Gao Z., Alarcon R.M., Ye J., Yun Z. (2009) Febs J 276(8) 2348-2548.

[62] Henegar C. (2008) Genome Biol.21;9(1):R14.

[63] Maasen J.A., Romijin J.A. (2007) Diabetologia, 50(10):2036-41.

[64] Rosner M., Fuchs C. (2009) Hum Mol Genet. 18(17):3298-310.

[65] Henegar C., Torjdman J. (2008) Genome Biol. 21;9(1):R14.

[66] Ye J. (2008) Int J Obes (Lond).;33(1):54-66.

[67] Carter J.C., Church F.C. (2009) PPAR Res., 345320, PMC2723729.

[68] Liu Y.Z., Pei Y.F., Liu J.F. (2009) PLoS One, 4(8):e6827, PMC2730014..

[69] Chung J., Nguyen A.K., Henstridge D.C., et al. (2008) Proc Natl Acad Sci USA. 105(5):1739-44.

[70] Kolehmainen M., Salopuro T. (2008) Int $J$ Obes., 32(2):292-303.

[71] Cao H., Yang C.S. (2008) PLoS One. ;3(7):e2820, PMC2474702.

[72] Lakshmipathy U., Brad L., Goff L. A., Rebecka Jörnsten, Ralph Graichen, Hart R.P. and Chesnut J.D. (2007) Stem Cells Dev. (6): 1003-1016.

[73] Tzur G., Levy A., Meiri E. (2008) PLoS One. 3(11):e3726.

[74] Ivey K.N., Muth A., Arnold J., King F.W., Yeh R.F., Fish J.E., Hsiao E.C., Schwartz R.J., Conklin B.R., Bernstein H.S., Srivastava D. (2008) Cell Stem Cell. 2(3):219-29.

[75] Wu L. , Belasco J.G. (2005) Mol Cell Biol. ;25(21):9198-208.

[76] Gao F.B. (2008) Trends Neurosci., 31(1):20-6.

[77] Cao H., Yang C.S. (2008) PLOS ONE, 3(7):e2820.

[78] Ren J., Jin P., Wang E., Marincola F.M., Stroncek D.F. (2009) J Transl Med. 7:20.

[79] Nimmo R.A., Slack F.J. (2009) Chromosoma.;118(4):405-18.

[80] Suh M.R., Lee Y., Kim J.Y. (2004) Dev Biol., 270(2):488-498.

[81] Houbaviy H.B., Murray M.F., Sharp P.A. (2003) Dev Cell, 5(2):351-358.

[82] Kohler A. and Hurt E. (2007) Nat Rev. Mol Cell Biol 10,761-773.

[83] Borchert G.M., Lanier W. and Davidson B.L. (2006) Nat Struct Mol Biol 13, 10971101.

[84] Bartel D.P. (2004) Cell 116(2): 281--297.

[85] Filipowicz W., Bhattacharyya S.N., Sonenberg N. (2008) Nat Rev Genet, 9:102-114. 
[86] Davis B.N., Hata A. (2009) Cell Commun Signal 10;7:18.

[87] Gregory B.D., O'Malley R.C., Lister R., Urich M.A., Tonti-Filippini J., Chen H., Millar A.H., Ecker J.R. (2008) Dev Cell. 14(6):854-66. .

[88] Ying Shao-Yao, Lin Shi-Lung (2009) Methods in molecular biology (Clifton, N.J.) 487():387-413.

[89] Rosenfeld N., Aharonov R., Meiri E., Rosenwald S., Spector Y., et al. (2008) Nat Biotechnol.;26:462-469.

[90] Yu S.L., Chen H.Y., Chang G.C., Chen C.Y., Chen H.W., et al.(2008) Cancer Cell.,13:48-57.

[91] Wyman S.K., Parkin R.K.; Duchaine T.F., and Slack F.J. (2009) Curr Oncol.;16(4):61-6, PMC272205. 